\title{
Imaged-Based Visual Servo Control for a VTOL Aircraft
}

\author{
Liying Zou, ${ }^{1,2}$ Huiguang Li, ${ }^{1}$ Wei Zhao, ${ }^{1}$ and Lei $\mathrm{Zhu}^{2}$ \\ ${ }^{1}$ College of Electrical Engineering, Yanshan University, Qinhuangdao 066004, China \\ ${ }^{2}$ College of Communication and Electronic Engineering, Qiqihar University, Qiqihar 161006, China \\ Correspondence should be addressed to Liying Zou; zouliying2007@126.com
}

Received 6 May 2017; Revised 5 August 2017; Accepted 23 August 2017; Published 28 September 2017

Academic Editor: Andrea Crivellini

Copyright (C) 2017 Liying Zou et al. This is an open access article distributed under the Creative Commons Attribution License, which permits unrestricted use, distribution, and reproduction in any medium, provided the original work is properly cited.

\begin{abstract}
This paper presents a novel control strategy to force a vertical take-off and landing (VTOL) aircraft to accomplish the pinpoint landing task. The control development is based on the image-based visual servoing method and the back-stepping technique; its design differs from the existing methods because the controller maps the image errors onto the actuator space via a visual model which does not contain the depth information of the feature point. The novelty of the proposed method is to extend the image-based visual servoing technique to the VTOL aircraft control. In addition, the Lyapunov theory is used to prove the asymptotic stability of the VTOL aircraft visual servoing system, while the image error can converge to zero. Furthermore, simulations have been also conducted to demonstrate the performances of the proposed method.
\end{abstract}

\section{Introduction}

Over the past few years, the control problem of vertical takeoff and landing (VTOL) aircraft has received an increased interest since they do not require strips for take-off or landing [1]. The main difficulty of VTOL aircraft control is that it is nonminimum phase and underactuated $[2,3]$. Many papers have considered the control of the VTOL aircraft using various methods [1-12]. In earlier works [5], approximate linearisation technique was proposed to design controllers for slightly nonminimum phase aircraft. In terms of the stabilization control, a nonlinear controller was presented in [6], which achieves asymptotic stability. In [7], an algorithm based on system-decomposition technique was addressed to deal with the output tracking problem of the VTOL aircraft. In particular, a nonlinear observer was designed and a backstepping technique was applied to achieve global output tracking of a VTOL aircraft in [8]. Reference [9] offered a new method for achieving global stability in a VTOL aircraft with bounded thrust input. Besides, [11] introduced a nonlinear state feedback controller using optimal control method. It should be pointed out that all of aforementioned works ignore the pinpoint landing problem.

Here, we focus on the pinpoint landing problem. Usually, the position of the VTOL aircraft is obtained by global positioning systems (GPS) [12]. However, the slow responses and obvious errors cannot guarantee the movement requirements of flexible VTOL aircraft. A camera is a candidate to obtain the current state, and visual servoing is a powerful tool to be applied in control field [13-17]. Visual servoing can be divided into two main classes [13]: position-based visual servoing (PBVS) and image-based visual servoing (IBVS). PBVS requires an accurate model of the object and is sensitive to image measurement errors. On the other hand, IBVS is less sensitive to those errors than PBVS, though it has its own problems. So, we use the IBVS method to solve the pinpoint landing problem. In addition, we adopt a new binocular visual servoing model addressed by [18], which does not need the depth information of the object and avoids the evaluation for depth information.

In this paper, we propose an image-based control scheme for the pinpoint landing problem of the VTOL aircraft in this paper. The main contribution is to extend the IBVS method to the VTOL aircraft control, and its advantage is to design the controller in image space. The outline of this paper is as follows: the system dynamics of the VTOL aircraft is introduced in Section 2. The binocular visual servoing model is formulated in Section 3. In Section 4, the methodology for designing the visual servoing system controller is developed and the stability analysis is given. Furthermore, the simulation results of the proposed control 
algorithm are shown in Section 5. Finally, the conclusions are drawn in Section 6.

\section{VTOL Aircraft Model}

In this paper, the VTOL aircraft described by [5] will be used to explore the use of the proposed method. In [5], the nominal mathematical model of the VTOL aircraft is

$$
\begin{aligned}
-\ddot{y} & =-u_{1} \sin \phi+\varepsilon u_{2} \cos \phi \\
-\ddot{z} & =u_{1} \cos \phi+\varepsilon u_{2} \sin \phi-g \\
\ddot{\phi} & =u_{2},
\end{aligned}
$$

where $(y, z)$ and $\phi$ denote, respectively, the position of the aircraft center of mass and roll angle, the controls $u_{1}$ and $u_{2}$ are the thrust and the rolling moment, respectively, $g>0$ is the gravitational acceleration, and $\varepsilon$ is a small constant coupling between the roll moment and the lateral force.

Here, the control objective is to design a robust control law so that the VTOL aircraft can stably land on the desired position, while keeping the internal dynamics stable.

For system (1), taking the input transformation,

$$
\left[\begin{array}{l}
u_{1} \\
u_{2}
\end{array}\right]=\left[\begin{array}{cc}
-\sin \phi & \varepsilon \cos \phi \\
\cos \phi & \varepsilon \sin \phi
\end{array}\right]^{-1}\left[\begin{array}{c}
v_{1} \\
v_{2}+g
\end{array}\right],
$$

where $v_{1}$ and $v_{2}$ are new inputs.

Then, the dynamics of the aircraft can be expressed as

$$
\begin{aligned}
-\ddot{y} & =v_{1} \\
-\ddot{z} & =v_{2} \\
\ddot{\phi} & =\frac{1}{\varepsilon} v_{1} \cos \phi+\frac{1}{\varepsilon} v_{2} \sin \phi+\frac{g}{\varepsilon} \sin \phi .
\end{aligned}
$$

$$
\left[\begin{array}{c}
\dot{x}_{1} \\
\dot{y}_{1} \\
\dot{x}_{2}
\end{array}\right]=\left[\begin{array}{cccccc}
\frac{x_{2}-x_{1}}{B} & 0 & \frac{x_{1}\left(x_{1}-x_{2}\right)}{f B} & \frac{x_{1} y_{1}}{f} & -\frac{f^{2}+x_{1}^{2}}{f} & y_{1} \\
0 & \frac{x_{2}-x_{1}}{B} & \frac{y_{1}\left(x_{1}-x_{2}\right)}{f B} & \frac{f^{2}+y_{1}^{2}}{f} & -\frac{x_{1} y_{1}}{f} & -x_{1} \\
\frac{x_{2}-x_{1}}{B} & 0 & \frac{x_{2}\left(x_{1}-x_{2}\right)}{f B} & \frac{y_{1} x_{2}}{f} & -\frac{f^{2}+x_{1} x_{2}}{f} & y_{1}
\end{array}\right] \cdot\left[\begin{array}{c}
v_{x} \\
v_{y} \\
v_{z} \\
\omega_{x} \\
\omega_{y} \\
\omega_{z}
\end{array}\right],
$$

where $\mathbf{m}=\left[\begin{array}{lll}x_{1} & y_{1} & x_{2}\end{array}\right]^{T} \in R^{3}$ denotes the image coordinate of the feature point, $B$ is the distance between the optical centers of two camera lens, and $f$ is the focal length; $\mathbf{V}_{c}=$ $\left[\begin{array}{llllll}v_{x} & v_{y} & v_{z} & \omega_{x} & \omega_{y} & \omega_{z}\end{array}\right]^{T} \in R^{6}$ is the velocity of the camera velocity in the world ordinate system. Then (7) can be rewritten as

\section{Binocular Visual Servoing Model}

According to [18], the binocular visual servoing model is described as

Let $w_{1}=-y, w_{2}=-\dot{y}, w_{3}=-z, w_{4}=-\dot{z}, w_{5}=\phi, w_{6}=$ $\dot{\phi}, \mathbf{w}=\left[\begin{array}{llllll}w_{1} & w_{2} & w_{3} & w_{4} & w_{5} & w_{6}\end{array}\right]^{T} ;$ we can obtain

$$
\begin{aligned}
& \dot{w}_{1}=w_{2} \\
& \dot{w}_{2}=v_{1} \\
& \dot{w}_{3}=w_{4} \\
& \dot{w}_{4}=v_{2} \\
& \dot{w}_{5}=w_{6} \\
& \dot{w}_{6}=\frac{1}{\varepsilon} v_{1} \cos w_{5}+\frac{1}{\varepsilon} v_{2} \sin w_{5}+\frac{g}{\varepsilon} \sin w_{5} ;
\end{aligned}
$$

where

where $J(\mathbf{m}) \in R^{3 \times 6}$ is the image Jacobian matrix of the feature point $\mathbf{m}$ and the model does not contain the depth information, thereby avoiding the estimation the depth information.

As the camera is mounted on the mass center of the VTOL aircraft, the following equation holds:

$$
\dot{\mathbf{m}}=J(\mathbf{m}) \mathbf{V}_{c},
$$

$$
\mathbf{V}_{c}=\left[\begin{array}{cccccc}
0 & \dot{y} & \dot{z} & \dot{\phi} & 0 & 0
\end{array}\right]^{T}=\mathbf{A} \mathbf{w},
$$


where

$$
\mathbf{A}=\left[\begin{array}{cccccc}
0 & 0 & 0 & 0 & 0 & 0 \\
0 & -1 & 0 & 0 & 0 & 0 \\
0 & 0 & 0 & -1 & 0 & 0 \\
0 & 0 & 0 & 0 & 0 & 1 \\
0 & 0 & 0 & 0 & 0 & 0 \\
0 & 0 & 0 & 0 & 0 & 0
\end{array}\right] .
$$

Substituting (9) into (8) yields

$$
\dot{\mathbf{m}}=\mathbf{K}(\mathbf{m}) \mathbf{w},
$$

where $\mathbf{K}(\mathbf{m})=J(\mathbf{m}) \mathbf{A}$.

For binocular visual model (8), it must be subject to the following assumptions:

(1) The intrinsic and extrinsic parameters of the camera are known.

(2) The object feature points are always in the camera field of view.

(3) The mass center of the aircraft is coincident with the origin of left camera coordinate.

\section{Controller Design of VTOL Aircraft Visual Servoing System}

In order to fulfill the pinpoint landing task, we design a controller using the back-stepping technique and the IBVS method in this section. The control objective is to make the image error converge to zero. Firstly, we establish the VTOL aircraft visual servoing system architecture. Then, we proceed the controller design for the VTOL aircraft visual servoing system.

Let the current image position of the object points $\mathbf{m}=y_{1}$ and $\mathbf{w}=y_{2}$; combining (5) with (11) yields the VTOL aircraft visual servoing system

$$
\begin{aligned}
& \dot{y}_{1}=\mathbf{K}\left(y_{1}\right) y_{2} \\
& \dot{y}_{2}=\mathbf{F}\left(y_{2}\right)+\mathbf{G}\left(y_{2}\right) \mathbf{v} .
\end{aligned}
$$

Let the desired image position of the object point $\mathbf{m}_{d}=$ $y_{1 d}$, and define the image error

$$
\mathbf{e}_{1}=y_{1 d}-y_{1} .
$$

Owing to $\mathbf{m}_{d}$ which is a constant, the time derivative of (13) will be

$$
\dot{\mathbf{e}}_{1}=\dot{y}_{1 d}-\dot{y}_{1}=-\mathbf{K}\left(y_{1}\right) y_{2} .
$$

Define the Lyapunov function

$$
V_{1}=\frac{1}{2} \mathbf{e}_{1}^{T} \mathbf{e}_{1} .
$$

Taking the time derivative of (15) yields

$$
\dot{V}_{1}=\mathbf{e}_{1}^{T} \dot{\mathbf{e}}_{1}=-\mathbf{e}_{1}^{T} \mathbf{K}\left(y_{1}\right) y_{2} .
$$

According to the back-stepping technique, choosing the virtual control,

$$
y_{2 d}=\mathbf{K}^{+}\left(y_{1}\right) \Lambda e_{1},
$$

where $\mathbf{K}^{+}\left(y_{1}\right)$ is the pseudoinverse matrix of $\mathbf{K}\left(y_{1}\right)$ and $\Lambda$ is a positive matrix to design latter.

Define the error

$$
\mathbf{e}_{2}=y_{2 d}-y_{2} .
$$

Substituting (17) and (18) into (16), it follows that

$$
\dot{V}_{1}=-\mathbf{e}_{1}^{T} \mathbf{K}\left(y_{1}\right)\left(y_{2 d}-\mathbf{e}_{2}\right)=-\mathbf{e}_{1}^{T} \Lambda \mathbf{e}_{1}+\mathbf{e}_{1}^{T} \mathbf{K}\left(y_{1}\right) \mathbf{e}_{2} .
$$

To guarantee the stability of the system, we choose the sliding mode variable as

$$
\mathbf{S}=\mathbf{e}_{2}+K_{1} \int_{0}^{t} \mathbf{e}_{2} d t
$$

where $K_{1}$ is a positive matrix to specify latter.

Define the Lyapunov function

$$
V_{2}=\frac{1}{2} \mathbf{S}^{\mathrm{T}} \mathbf{S}
$$

Taking the time derivative of (21) yields

$$
\begin{aligned}
\dot{V}_{2} & =\mathbf{S}^{\mathrm{T}} \dot{\mathbf{S}}=\mathbf{S}^{\mathbf{T}}\left(\dot{\mathbf{e}}_{2}+K_{1} \mathbf{e}_{2}\right)=\mathbf{S}^{\mathbf{T}}\left(\dot{y}_{2 d}-\dot{y}_{2}+K_{1} \mathbf{e}_{2}\right) \\
& =\mathbf{S}^{\mathbf{T}}\left[\dot{y}_{2 d}-\mathbf{F}\left(y_{2}\right)-\mathbf{G}\left(y_{2}\right) \mathbf{v}+K_{1} \mathbf{e}_{2}\right] .
\end{aligned}
$$

In conclusion, we design the sliding control law as follows:

$$
\mathbf{v}=\mathbf{G}^{+}\left(y_{2}\right)\left[\dot{y}_{2 d}-\mathbf{F}\left(y_{2}\right)+K_{1} \mathbf{e}_{2}+M \mathbf{S}\right],
$$

where $M$ is a positive matrix to specify latter.

Substituting (23) into (22) we have

$$
\dot{V}_{2}=-\mathbf{S}^{\mathrm{T}} \mathbf{M S}<0 .
$$

Therefore, it is easy to conclude that $\lim _{t \rightarrow \infty} \mathbf{S}=0$, which can deduce $\mathbf{e}_{2}=-K_{1} \int_{0}^{t} \mathbf{e}_{2} d t$; that is to say, $\dot{\mathbf{e}}_{2}=-K_{1} \mathbf{e}_{2}$. Seeing that $K_{1}>0$, it follows that $\lim _{t \rightarrow \infty} \mathbf{e}_{2}=0$; that is, $\lim _{t \rightarrow \infty} y_{2}=y_{2 d}=\mathbf{K}^{+}\left(y_{1}\right) \Lambda e_{1}$. Owing to $\dot{y}_{1}=\mathbf{K}\left(y_{1}\right) y_{2}$, we obtain $\dot{y}_{1}=\mathbf{K}\left(y_{1}\right) y_{2 d}=\Lambda \mathbf{e}_{1}$; that is, $\lim _{t \rightarrow \infty} \mathbf{e}_{1}=0$. In addition, considering $\lim _{t \rightarrow \infty} y_{2}=\mathbf{K}^{+}\left(y_{1}\right) \Lambda e_{1}$, we can conclude that $\lim _{t \rightarrow \infty} y_{2}=0$ and $\lim _{t \rightarrow \infty} \mathbf{w}=0$; that is, $w_{1} \rightarrow 0, w_{2} \rightarrow 0, w_{3} \rightarrow 0, w_{4} \rightarrow 0, w_{5} \rightarrow 0, w_{6} \rightarrow 0$. The VTOL aircraft fulfills the landing task.

Further, to sum up main results of this paper, we provide the following theorems.

Theorem 1. Consider the VTOL aircraft visual servoing system described by (12); if one chooses the controller given in (23), the closed-loop system is asymptotically stable. 

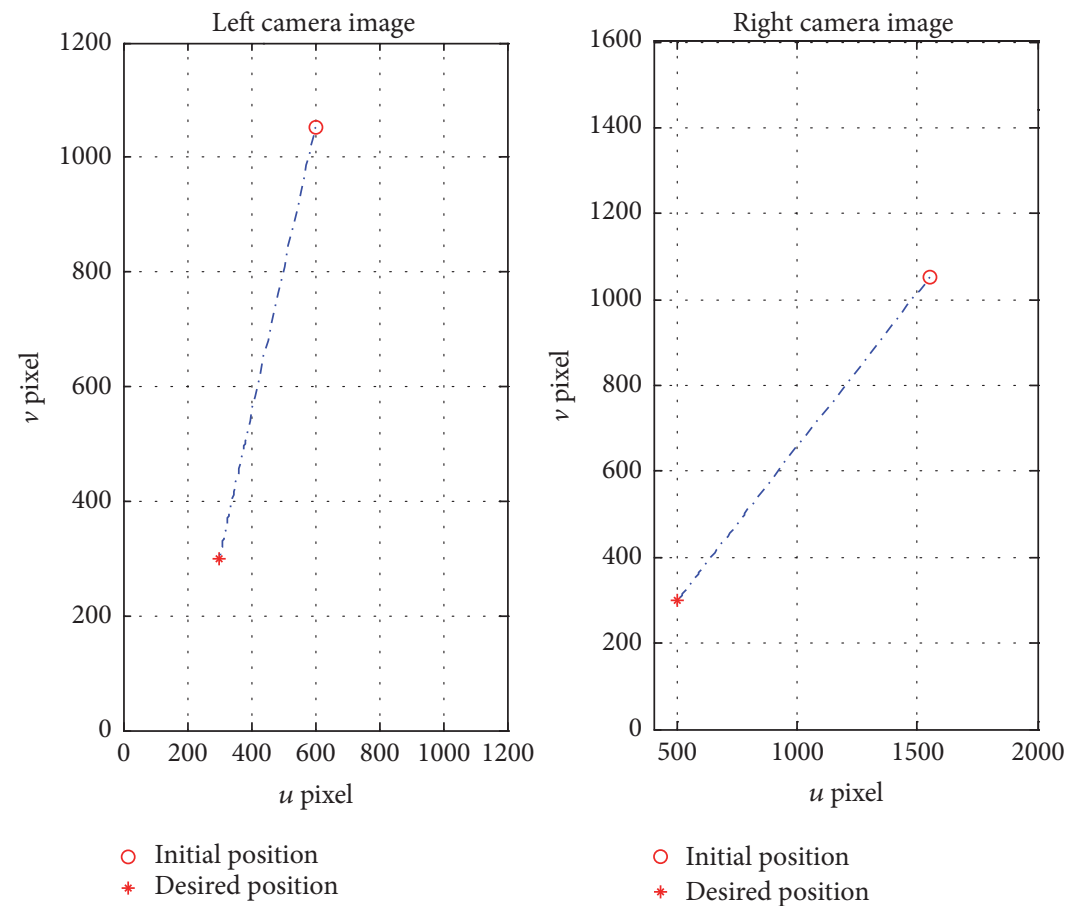

FIGURE 1: Trajectories of the feature point in the image plane.
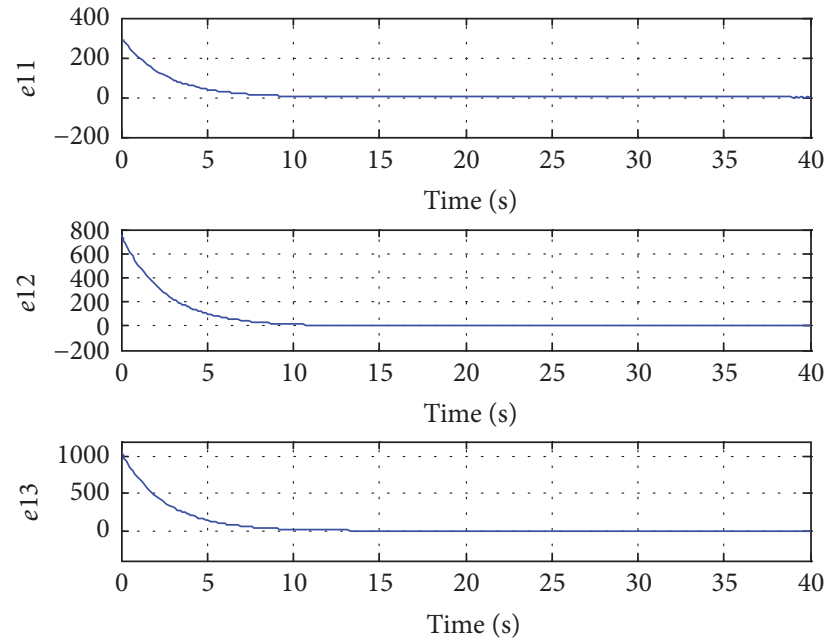

Figure 2: Image error curve.

\section{Simulation Results}

In this section, we verify the effectiveness of the control method in this paper by computer simulation results as shown in Figures 1-5. We consider a VTOL aircraft governed by (12), and we consider $g=9.8 \mathrm{~m} / \mathrm{s}^{2}$. The image coordinates of the feature point is obtained by an onboard camera which is centered at $(256,256)$ (pixels) and having a ratio focal length to pixel size equal to 900 . The coupling parameter is selected as $\varepsilon=0.5$. In addition, we assume that the object feature points are always in the camera field of view. The initial states are taken as $\mathbf{w}_{0}=\left[\begin{array}{llllll}3.5 & 0.01 & 3.0 & 0.01 & 0.05 & 0\end{array}\right]^{T}$ and the
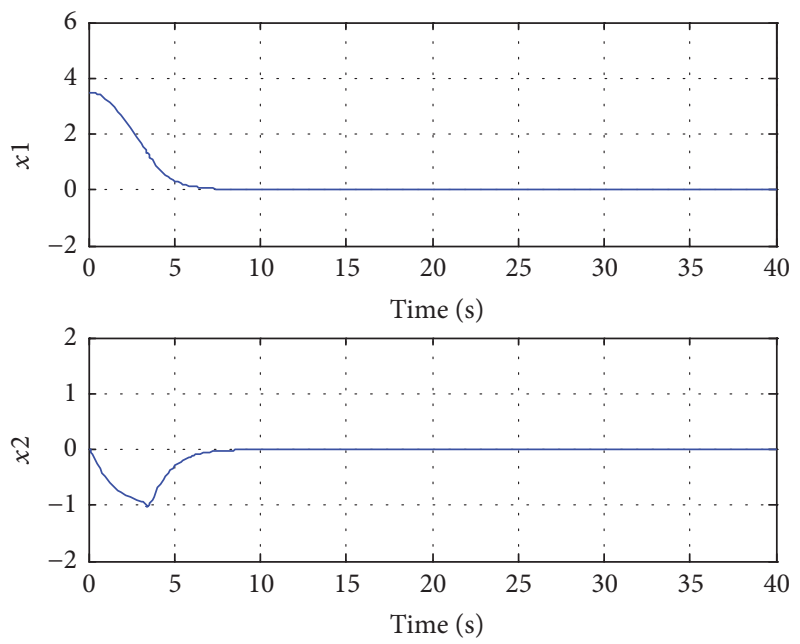

FIGURE 3: The horizontal position and velocity.

desired states are given as $\mathbf{w}_{\mathrm{d}}=\left[\begin{array}{llllll}0.1 & 0 & 0.3 & 0 & 0 & 0\end{array}\right]^{T}$. The control gains are chosen as

$$
\begin{aligned}
M & =10 \operatorname{diag}(1,1,1,1,1,1), \\
K_{1} & =20 \operatorname{diag}(1,1,1,1,1,1), \\
\Lambda & =5 \operatorname{diag}(1,1,1) .
\end{aligned}
$$

Figure 1 illustrates the trajectories of the feature point in image plane, which shows that the aircraft converges to the desired landing position. As shown in Figure 2, the image errors asymptotically converge to zero in a short time, which 

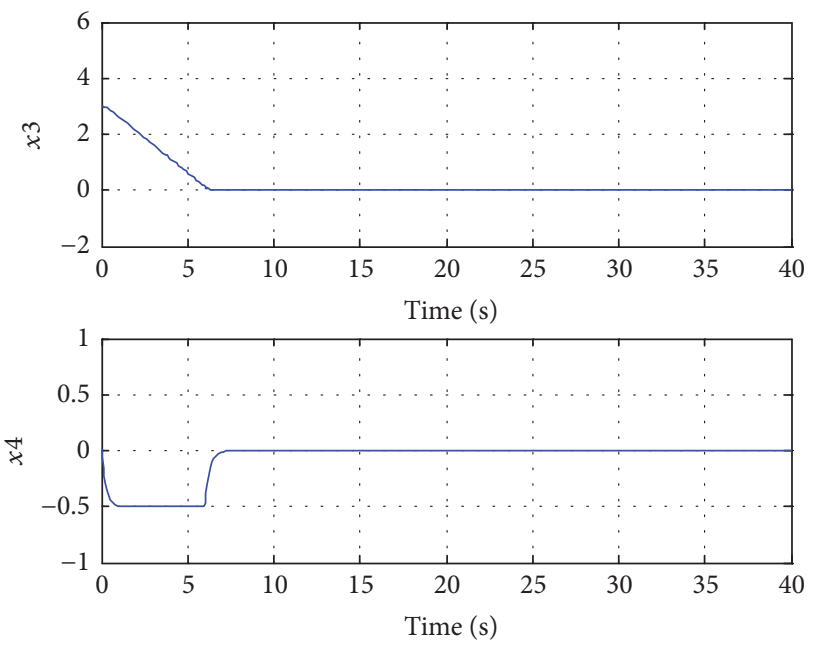

Figure 4: The vertical position and velocity.
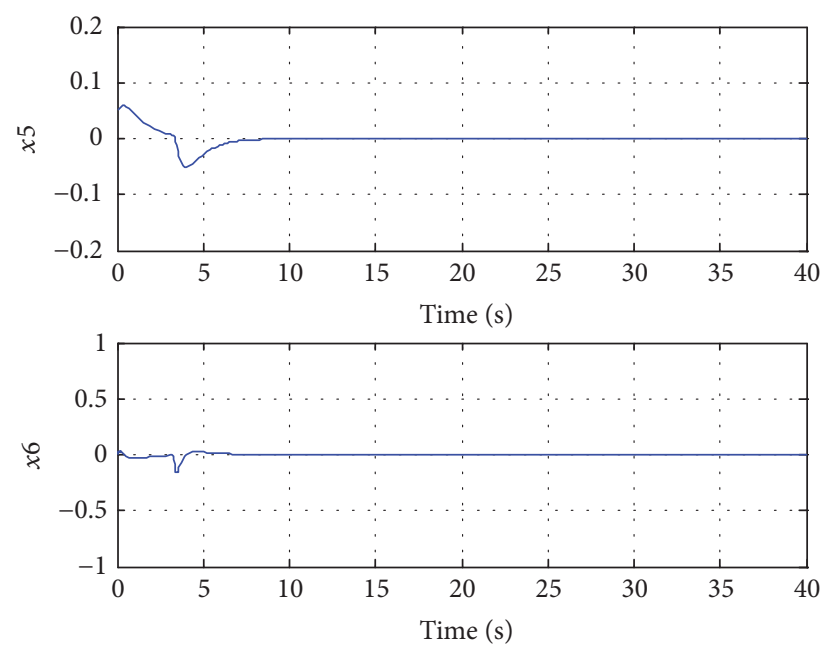

FIGURE 5: The roll angle and angle velocity.

means that the aircraft fulfill the pinpoint landing task rapidly. In addition, Figures 3 and 4 show the transient responses of aircraft in horizontal direction and vertical direction, from which we can see that the aircraft system has a fast response and good accuracy.

From Figure 5, it can be seen that the roll angle and angle velocity converge to zero in a short time, which imply that the internal dynamics keep stable. These results demonstrate that the proposed method in this paper behaves well.

In what follows, we will compare our results with previous results from [19], whose simulation results are shown in Figures 6-8. From Figures 6 and 7, it can be easily seen that the transient responses get close to the zero within $15 \mathrm{~s}$, which have a slow convergence rate in contrast with our results in Figures 3 and 4. Moreover, Figure 8 shows the roll angle and angle velocity curve; it is obvious that the roll angle and angle velocity become close to the zero in a short time but take a longer time to settle to the zero compared to our corresponding results in Figure 5.
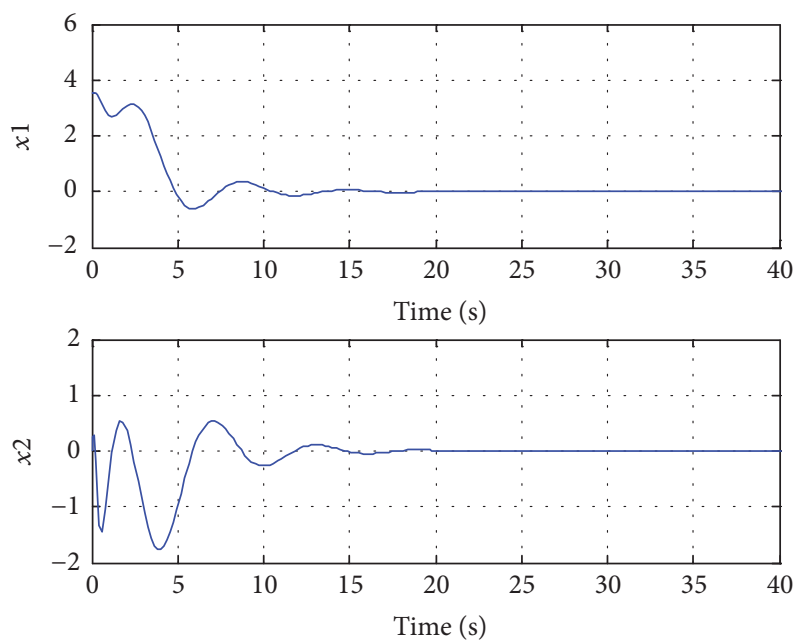

Figure 6: The horizontal position and velocity.
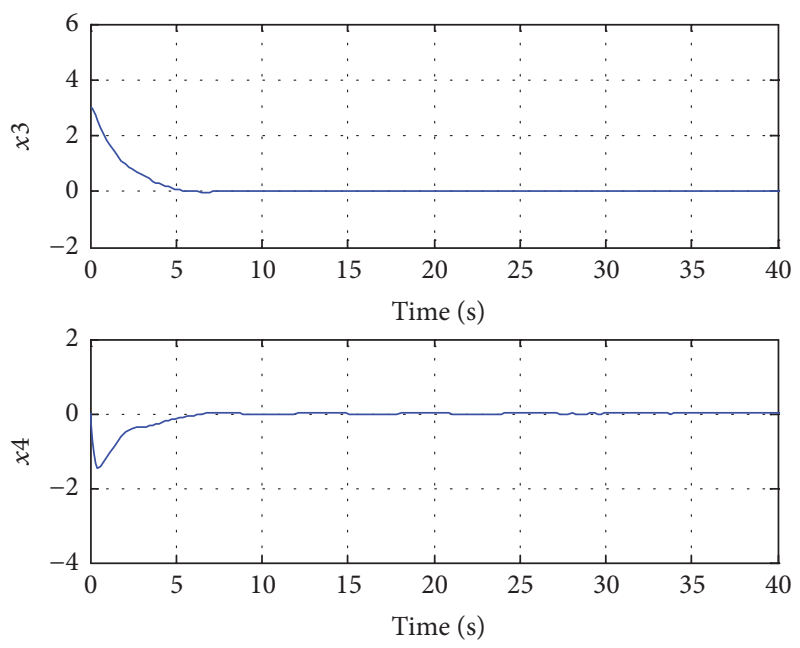

FIGURE 7: The vertical position and velocity.

From above comparative analysis, it can be conclude that our method is effective and feasible.

\section{Conclusion}

The pinpoint landing control problem of VTOL aircraft has been discussed in this paper. The control development is based on the image-based visual servoing method and the back-stepping technique. Firstly, on the basis of the binocular vision model addressed by [18], we construct the VTOL aircraft visual servoing system architecture, which does not contain the depth information of the feature point and avoids the depth information evaluation. Then, a controller is developed by using the back-stepping technique and sliding mode method. Moreover, it has been proved that the overall closed-loop system is asymptotically stable, while the image error converges to zero. Finally, simulation results show satisfactory response of the presented approach for pinpoint landing task of the VTOL aircraft. 

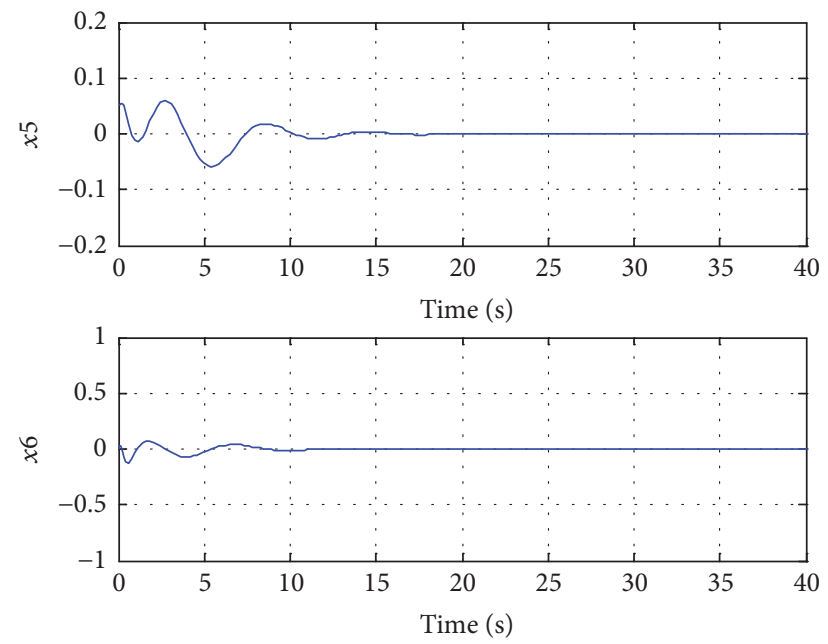

Figure 8: The roll angle and angle velocity.

\section{Conflicts of Interest}

The authors declare that they have no conflicts of interest.

\section{Acknowledgments}

This work was supported by Natural Science Foundation of China (61501275) and Natural Science Foundation of Heilongjiang Province (QC2015073).

\section{References}

[1] S. Gupte, P. I. T. Mohandas, and J. M. Conrad, "A survey of quadrotor unmanned aerial vehicles," in Proceedings of the IEEE Southeastcon, pp. 1-6, Orlando, Fla, USA, March 2012.

[2] S. A. Al-Hiddabi and N. H. McClamroch, "Output tracking for nonlinear non-minimum phase VTOL aircraft," in Proceedings of the 37th IEEE Conference on Decision and Control (CDC '98), pp. 4573-4578, Tampa, Fla, USA, December 1998.

[3] R. Olfati-Saber, "Global configuration stabilization for the VTOL aircraft with strong input coupling," IEEE Transactions on Automatic Control, vol. 47, no. 11, pp. 1949-1952, 2002.

[4] B. Zhu, X. Wang, and K.-Y. Cai, "Approximate trajectory tracking of input-disturbed PVTOL aircraft with delayed attitude measurements," International Journal of Robust and Nonlinear Control, vol. 20, no. 14, pp. 1610-1621, 2010.

[5] J. Hauser, S. Sastry, and G. Meyer, "Nonlinear control design for slightly nonminimum phase systems: application to V/STOL aircraft," Automatica, vol. 28, no. 4, pp. 665-679, 1992.

[6] C.-S. Huang and K. Yuan, "Output tracking of a non-linear non-minimum phase PVTOL aircraft based on non-linear state feedback control," International Journal of Control, vol. 75, no. 6, pp. 466-473, 2002.

[7] S. A. Al-Hiddabi and N. H. McClamroch, "Tracking and maneuver regulation control for nonlinear nonminimum phase systems: application to flight control," IEEE Transactions on Control Systems Technology, vol. 10, no. 6, pp. 780-792, 2002.

[8] X. Wang, J. Liu, and K.-Y. Cai, "Tracking control for a velocitysensorless VTOL aircraft with delayed outputs," Automatica, vol. 45, no. 12, pp. 2876-2882, 2009.
[9] A. Ailon, "A control for autonomous VTOL aircraft with restricted inputs," in Proceedings of the 17th IEEE Mediterranean Conference on Control and Automation, pp. 1569-1574, Thessaloniki, Greece, 2009.

[10] K. D. Do, Z. P. Jiang, and J. Pan, "On global tracking control of a VTOL aircraft without velocity measurements," IEEE Transactions on Automatic Control, vol. 48, no. 12, pp. 2212-2217, 2003.

[11] F. Lin, W. Zhang, and R. D. Brandt, "Robust hovering control of a PVTOL aircraft," IEEE Transactions on Control Systems Technology, vol. 7, no. 3, pp. 343-351, 1999.

[12] X. Wang, "Takeoff/landing control based on acceleration measurements for VTOL aircraft," Journal of the Franklin Institute, vol. 350, no. 10, pp. 3045-3063, 2013.

[13] F. Janabi-Sharifi, L. Deng, and W. J. Wilson, "Comparison of basic visual servoing methods," IEEE/ASME Transactions on Mechatronics, vol. 16, no. 5, pp. 967-983, 2011.

[14] T. Hamel and R. Mahony, "Visual servoing of an under-actuated dynamic rigid-body system: an image-based approach," IEEE Transactions on Robotics and Automation, vol. 18, no. 2, pp. 187198, 2002.

[15] E. Altuğ, J. P. Ostrowski, and C. J. Taylor, "Control of a quadrotor helicopter using dual camera visual feedback," The International Journal of Robotics Research, vol. 24, no. 5, pp. 329-341, 2005.

[16] T. Hamel and R. Mahony, "Image based visual servo control for a class of aerial robotic systems," Automatica, vol. 43, no. 11, pp. 1975-1983, 2007.

[17] A. Astolfi, L. Hsu, M. S. Netto, and R. Ortega, “Two solutions to the adaptive visual servoing problem," IEEE Transactions on Robotics and Automation, vol. 18, no. 3, pp. 387-392, 2002.

[18] H. Li, M. Jin, and L. Zou, "A new binocular stereo visual servoing model," in Proceedings of the Pacific-Asia Workshop on Computational Intelligence and Industrial Application (PACIIA '08), pp. 461-465, Wuhan, China, December 2008.

[19] M. T. Frye, S. Ding, C. Qian, and S. Li, "Fast convergent observer design for output feedback stabilisation of a planar vertical takeoff and landing aircraft," IET Control Theory \& Applications, vol. 4, no. 4, pp. 690-700, 2010. 


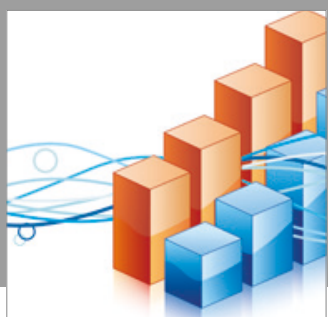

Advances in

Operations Research

vatersals

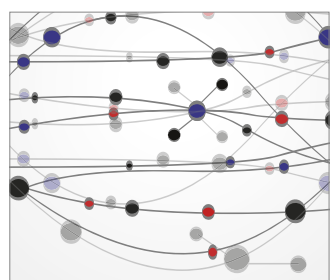

\section{The Scientific} World Journal
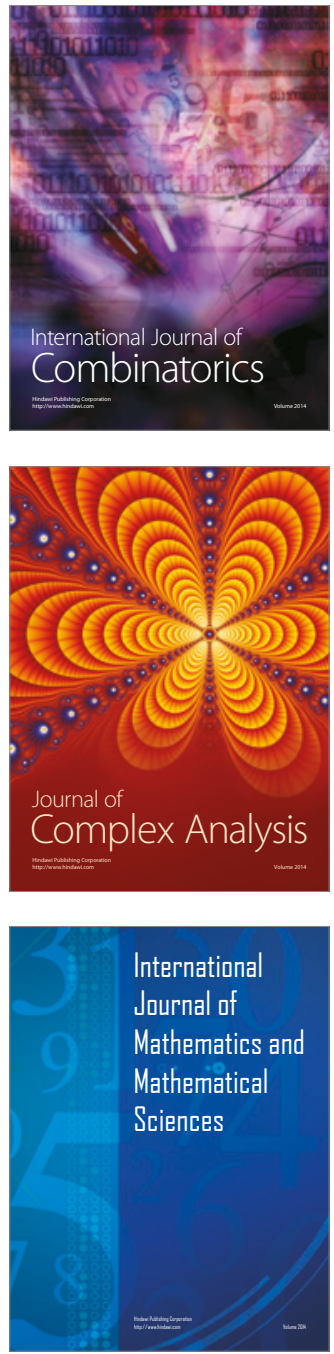
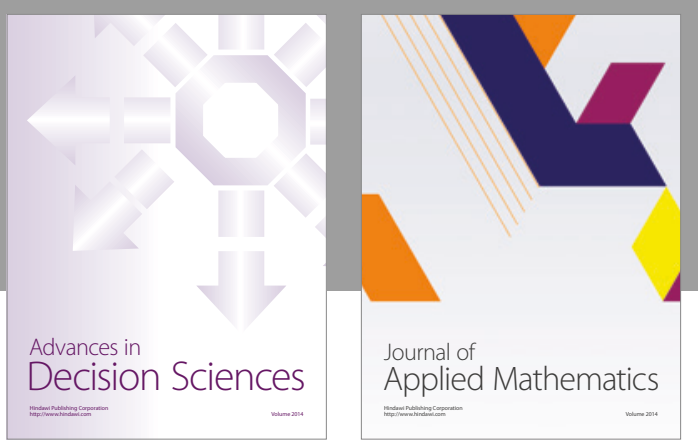

Algebra

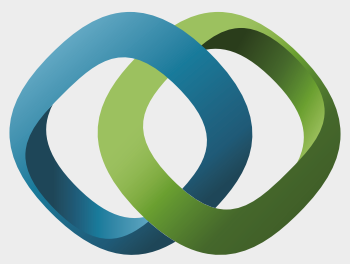

\section{Hindawi}

Submit your manuscripts at

https://www.hindawi.com
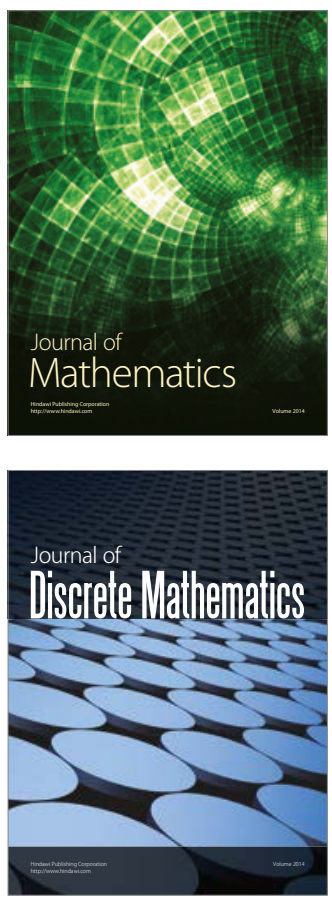

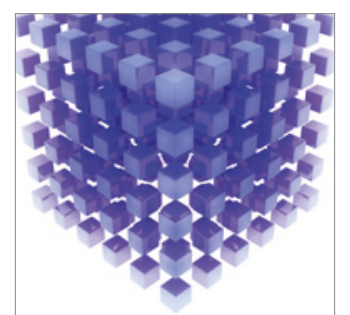

Mathematical Problems in Engineering
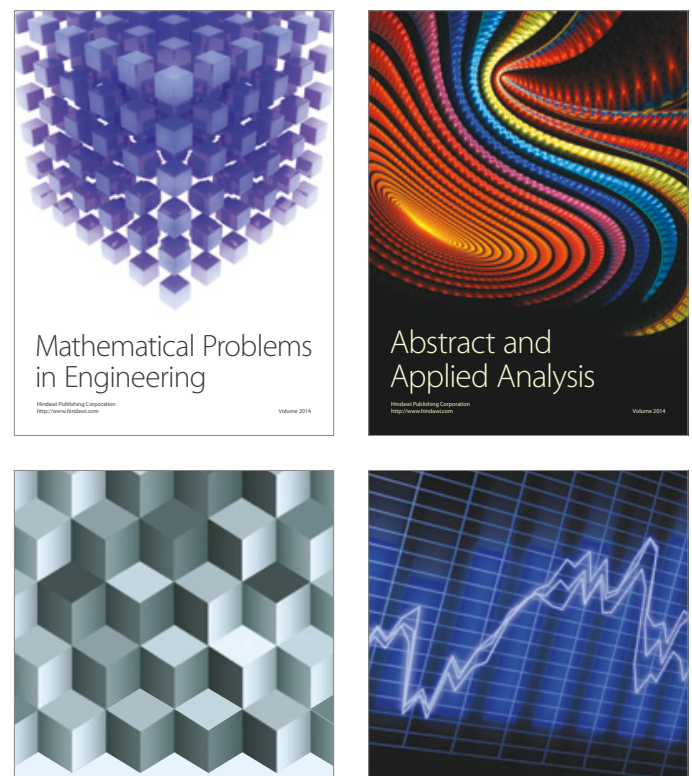

Journal of

Function Spaces

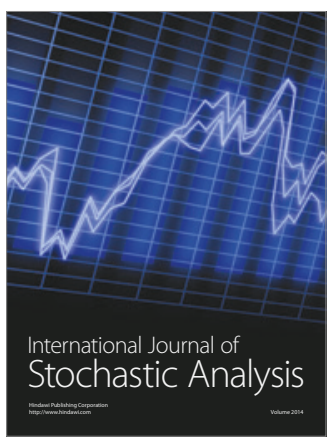

Probability and Statistics
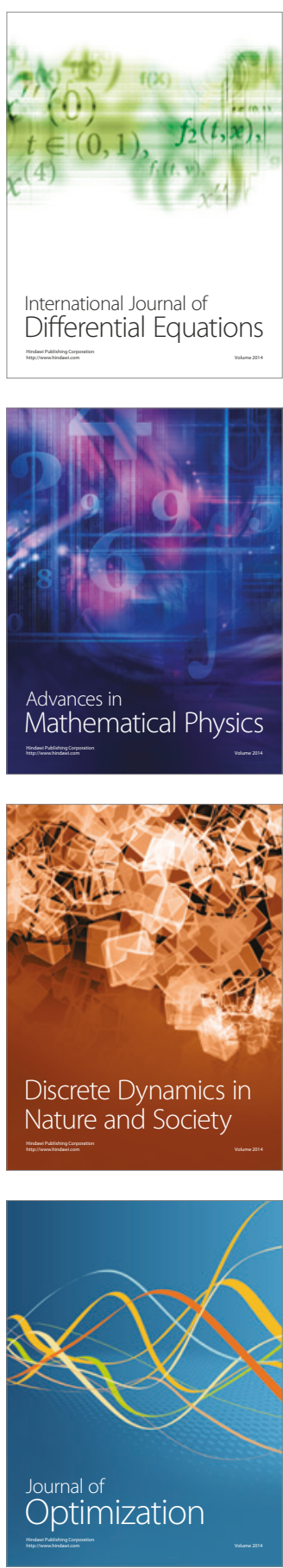